Geometrical Properties of a "Snow-Flake" Divertor

D. D. Ryutov

February 8, 2007

Physics of Plasmas 
This document was prepared as an account of work sponsored by an agency of the United States Government. Neither the United States Government nor the University of California nor any of their employees, makes any warranty, express or implied, or assumes any legal liability or responsibility for the accuracy, completeness, or usefulness of any information, apparatus, product, or process disclosed, or represents that its use would not infringe privately owned rights. Reference herein to any specific commercial product, process, or service by trade name, trademark, manufacturer, or otherwise, does not necessarily constitute or imply its endorsement, recommendation, or favoring by the United States Government or the University of California. The views and opinions of authors expressed herein do not necessarily state or reflect those of the United States Government or the University of California, and shall not be used for advertising or product endorsement purposes. 


\title{
Geometrical properties of a "snow-flake" divertor
}

\author{
D.D. Ryutov \\ Lawrence Livermore National Laboratory, Livermore, CA 94551
}

\begin{abstract}
Using a simple set of poloidal field coils, one can reach the situation where the null of the poloidal magnetic field in the divertor region is of a second order, not of the first order as in the usual X-point divertor. Then, the separatrix in the vicinity of the null-point splits the poloidal plane not into four sectors, but into six sectors, making the whole structure looking like a snow-flake (whence a name, [1]). This arrangement allows one to spread the heat load over much broader area than in the case of a standard divertor. A disadvantage of this configuration is in that it is topologically unstable, and, with the current in the plasma varying with time, it would switch either to the standard X-point mode, or to the mode with two $\mathrm{X}$-points close to each other. To avoid this problem, it is suggested to have a current in the divertor coils by roughly $5 \%$ higher than in an "optimum" regime (the one where a snow-flake separatrix is formed). In this mode, the configuration becomes stable and can be controlled by varying the current in the divertor coils in concert with the plasma current; on the other hand, a stron flaring of the scrape-off layer still remains in force. Geometrical properties of this configurations are analyzed for a simple model. Potential advantages and disadvantages of this scheme are discussed.
\end{abstract}

Reducing heat loads on the divertor plates would improve the overall performance of a future reactor-tokamak (e.g. [1]). There are various ideas regarding the means for reaching this goal [1]. We here concentrate on the approach that would use geometrical effects for a stronger flaring of the poloidal field in the divertor. An elegant solution of this type has been recently proposed by M. Kotschenreuther et al [2], in the form of what they call the X-divertor, where the poloidal flux experiences an additional expansion already after entering the divertor zone. In our brief communication, we consider an approach where the null of the poloidal magnetic field in the divertor region is second order, not first order as in the usual X-point divertor (the term X-divertor should not be confused with the X-point divertor; by the latter we mean a standard X-point configuration as used in a number of tokamaks, e.g., [3-6]). Then, as we show below, the separatrix in the vicinity of the null-point splits the poloidal plane not into four sectors, but into six sectors (Fig. 1 a), making the whole structure look like a snow-flake (whence the name). Because the null of the poloidal field is now of a higher order than in a standard X-point configuration, the magnetic flux threading the scrape-off-layer (SOL) expands near the null-point much stronger than in a standard situation. This gives a hope that a snow-flake divertor may indeed be helpful in reducing the heat load either on its own, or in combination with other techniques.

To quantify the properties of this divertor, we use a simple model where we consider a "rectified" (i.e., straight) tokamak and where the plasma current is imitated just by a single straight conductor (Fig. 1). This allows us to make a quick scoping study and identify key advantages and disadvantages of the proposed configuration. Later in the paper, we show that the basic properties of the divertor remain intact when one considers an arbitrary distribution of the plasma current and includes the toroidicity effect.

It turns out that the desired result can be reached by using just two divertor coils properly spaced and with a properly chosen current (Fig. 1). Let us denote the plasma 
current by $I$, whereas the total current in two identical "divertor" conductors by $I_{d}$. Let the distance between the null-point (which, we assume, is situated in the origin, $x=z=0$ ) and the "plasma" conductor be $a$, the distance between the symmetrically-situated divertor conductors be $d$, and both of them being below the null-point by $b$ (Fig. 1).

It is easy to check that the field is indeed zero at $x=z=0$ if the condition

$$
I_{d}=I\left(b+\frac{d^{2}}{4 b}\right)
$$

holds. The condition that the linear terms in the expansion of $B_{x}$ and $B_{z}$ over $x$ and $z$ near the null-point are also zero, with the total current in two conductors determined from (1), leads to the constraint

$$
d=2 b \sqrt{\frac{a+b}{a-b}},
$$

this, in turn, meaning (by virtue of Eq. (1)) that the total current in the divertor coils must be equal to

$$
I_{d}=I_{d 0} \equiv I \frac{2 b}{a-b}
$$

The shape of the separatrix and two nearby flux surfaces for $b=0.3 a$, is shown in Fig. 1. Note a very strong expansion of SOL near the null-point. This is due to the fact that the magnetic field in this zone scales as square of the distance from the null-point (not linearly as in a standard divertor). If one denotes the SOL thickness near the equatorial plane by $\Delta_{0}$, then the SOL thickness in the snow-flake divertor near the nullpoint, $\Delta$, will scale as $\Delta \sim \Delta_{0}\left(b / \Delta_{0}\right)^{1 / 3}$, whereas in a usual X-point divertor one would have $\Delta \sim \Delta_{0} \ln \left(b / \Delta_{0}\right)$.

According to Eq. (2), the coils are situated at a distance $b \sqrt{2 a /(a-b)}$ from the null-point. In a reactor, the divertor coils should be placed outside the radiation shield, i.e., they cannot be situated too close to the plasma. As a representative value of $b$, we take $b=0.3 a$; then the coils will be situated at a distance $\sim 0.5 a \sim 2.5 \mathrm{~m}$ from the nullpoint. This seems to be sufficiently far, given that the shield thickness is $\sim 1 \mathrm{~m}$ (e.g., [6]). The current per divertor coil, according to Eq. (3) will be $\sim 0.45 I$, with the total current $\sim$ 0.9I. For non-radioactive experimental facilities one can, of course, consider much more compact snow-flake divertors, with a smaller ratio $b / a$.

A disadvantage of a snow-flake configuration is its topological instability: if the plasma (or divertor) current do not exactly satisfy Eq. (3), the configuration in the vicinity of the X-point becomes either an X-point configuration (Fig. 2a), for the divertor current higher than needed, or a double-X-point configuration (Fig. 2b) for the divertor current smaller than needed. The position of the strike point and the overall structure of the divertor plasma change substantially for a small variation of a plasma current if the initial current is exactly $I_{d 0}$.

We suggest making the configuration more robust by operating at a divertor current somewhat higher than Eq. (3). As we show below, in this case, minor variations of the plasma current and divertor current do not cause any substantial changes of the configuration and, at the same time, a strong flux expansion still occurs (if the current mismatch is modest).

The opposite possibility, namely, running the divertor at the current that is somewhat smaller than optimum, also provides the robustness to the configuration and 
significant flux expansion. However, as one can see from Fig. 2b, in this case the divertor region contacts the plasma core not in one point but rather along a line (CD, Fig. 2b). Although the possible effect of the line contact is at present not quite clear, we prefer to stay within a more customary geometry of Fig. 2a. One obvious concern regarding the configuration of Fig. $2 b$ is that the line contact may have an adverse effect on impurity penetration from divertor to the plasma core. We will call the configuration of Fig. 2a (with the divertor current somewhat higher than $I_{d 0}$ ) "snow-flake-plus", and the configuration of Fig. $2 b$ "snow-flake-minus."

For a more detailed analysis of the magnetic field structure in the divertor region, one can use an expansion of the magnetic field near the point $x=z=0$. Some rather lengthy algebra leads one to the following result for the normalized magnetic field ( $\hat{\boldsymbol{B}}=(a c / 2 I) \boldsymbol{B}$, the CGS system is used):

$$
\begin{aligned}
& \hat{B}_{x}=1-\frac{I_{d}}{I} \frac{4 a b}{4 b^{2}+d^{2}}+\frac{z}{a}\left[1+4 \frac{I_{d}}{I} \frac{a^{2}\left(4 b^{2}-d^{2}\right)}{\left(4 b^{2}+d^{2}\right)^{2}}\right]+\frac{z^{2}-x^{2}}{a^{2}}\left[1-4 \frac{I_{d}}{I} \frac{a^{2}\left(4 b^{2}-d^{2}\right)}{\left(4 b^{2}+d^{2}\right)^{2}}\right] \\
& \hat{B}_{z}=\frac{x}{a}\left[1+4 \frac{I_{d}}{I} \frac{a^{2}\left(4 b^{2}-d^{2}\right)}{\left(4 b^{2}+d^{2}\right)^{2}}\right]+\frac{2 x z}{a^{2}}\left[1-4 \frac{I_{d}}{I} \frac{a^{2}\left(4 b^{2}-3 d^{2}\right)}{\left(4 b^{2}+d^{2}\right)^{2}}\right]
\end{aligned}
$$

If the parameters exactly correspond to the snow-flake configuration, i.e., conditions (2) and (3) hold, only quadratic terms survive, and we recover a configuration shown in the inset in Fig. 1. If, however, as we are now assuming, the divertor current is somewhat higher than $I_{d 0}$,

$$
I_{d}=I_{d 0}(1+\varepsilon)
$$

whereas the position of the current rods remains untouched (i.e., condition (2) still holds), a switch to configuration of Fig. 2a occurs. Indeed, Eqs. (5), (6), with only dominant terms retained, become:

$$
\begin{aligned}
& \hat{B}_{x}=-\varepsilon-\varepsilon \frac{z}{a}+\frac{z^{2}-x^{2}}{a b} \frac{a+b}{b} \\
& \hat{B}_{z}=\varepsilon \frac{x}{a}+\frac{2 x z}{a b} \frac{a+b}{b}
\end{aligned}
$$

One can show that, for $\varepsilon \neq 0$, the separation $A B$ (Fig 2a) between the null and the lower branch of the separatrix scales as $\varepsilon^{1 / 2}, A B \approx 2.7 a b \sqrt{\varepsilon /(a+b)}$. The $\varepsilon^{1 / 2}$ scaling is favorable in the sense that, as soon as we have chosen some value of $\varepsilon$, say $\varepsilon=0.05$, the further variations with respect to this value (say, to $\varepsilon=0.07$ or to $\varepsilon=0.03$ ) do not lead to a significant change of the geometry in the vicinity of the null-point. This conclusion is illustrated by Fig. 3 .

Compare now the flux expansion properties of the snow-flake-plus divertor, Fig. 2a, where a null-point is "almost" the second order point, and of a more "canonical" divertor, where the null point is decisively of the first order. For the first of them we use the following parameters: $b=0.3, I_{d}=1.05 I_{d 0}$. For a "canonical" divertor, we use a configuration shown by light line in Fig. 2a. It corresponds to a single divertor conductor situated at a distance $b=0.6 a$ below the null point, with the current $I_{d}=0.65 I$. In the zone above the X-point the two look quite similar. In Fig. 4 a blow-up of the zone near the null-point is shown. Thin lines show the position of flux surfaces corresponding to the SOL boundary, which in both cases is assumed to be situated at the distance of $0.002 a$ 
from the separatrix at the equatorial plane (i.e., at the distance $1 \mathrm{~cm}$ for $a=500 \mathrm{~cm}$ ). One sees that, indeed, in the case of a snow-flake divertor the width of the SOL near the Xpoint is approximately 2.5 times greater than in the case of a "standard" divertor.

Because of a larger flux expansion, the connection length between the equatorial plane and the vicinity of the null-point becomes larger than for the "standard" divertors. For the flux surfaces not-too-close to the separatrix,

$$
\Delta_{0}>\varepsilon^{3 / 2} b^{2} / a,
$$

the connection length scales as $L \sim\left(a B_{T} / B_{P}\right)\left(a^{2} / b \Delta_{0}\right)^{1 / 3}$, where $B_{T}$ and $B_{P}$ are the toroidal and poloidal field near the equatorial plane. For smaller $\Delta_{0}$, the standard logarithmic dependence takes over. The larger connection length means the longer plasma transit time between the equatorial plane and the divertor thereby allowing for a stronger plasma cooling by radiative losses.

Also, for the flux surfaces satisfying condition (9) the squeezing of the flux tubes on their way from the equatorial plane to the plane where the null-point is situated (see Ref. [7]), becomes stronger than for the canonical X-point divertor. The squeezing can be conveniently characterized by the parameter called elongation $E$, the ratio of the major semi-axis of a flux-tube in the vicinity of the null-point, to the initial flux-tube radius near the equator. In the snow-flake-plus divertor, for the flux surfaces satisfying Eq. (9), the elongation is $\sim\left(b / \Delta_{0}\right)^{3 / 4}$, whereas for the standard divertor it is $\sim\left(b / \Delta_{0}\right)^{1 / 2}$. This stronger squeezing causes a more complete decoupling of the plasma perturbations in the divertor and the main SOL. Therefore, the operation space for the techniques based on the artificial stirring of the plasma in the divertor legs [8] (for the purpose of stronger spreading of the heat flux) becomes wider.

Now we show that the formation of the second-order null-point is compatible with a general toroidal geometry, and with an arbitrary distribution of the plasma current. The only assumption that we make is that the plasma in the divertor region has a low beta, and the magnetic field there can be considered as curl-free. We use a cylindrical coordinate system, $r, \theta$, $z$, with the radius $\mathrm{r}$ directed along the major radius, $\theta$ directed along the toroidal angle, and $z$ directed along the geometrical axis. The poloidal magnetic field can be conveniently characterized by the function $\Psi \equiv r A_{\theta}$, with $A_{\theta}$ being the toroidal component of the vector potential. One has:

$$
B_{r}=-\frac{1}{r} \frac{\partial \Psi}{\partial z} ; B_{z}=\frac{1}{r} \frac{\partial \Psi}{\partial r}
$$

The condition $\operatorname{div} \boldsymbol{B}=0$ is automatically satisified. In the curl-free area which the divertor is to a high accuracy, the function satisfies also the equation:

$$
r \frac{\partial}{\partial r}\left(\frac{1}{r} \frac{\partial \Psi}{\partial r}\right)+\frac{\partial^{2} \Psi}{\partial z^{2}}=0
$$

The equation of the poloidal field line is, obviously, $\Psi=$ const.

Assume that we are interested in creating a second-order null at some specific point. We choose the origin of $z$ in this point, and denote its radial coordinate by $\mathrm{r}_{0}$. Instead of radius $\mathrm{r}$, it is convenient to introduce a new variable $x$, via $r=r_{0}+x$. Let the poloidal magnetic field created at this point by the plasma current be $B_{r 0}$ and $B_{z 0}$. In the expansion of $\Psi$ over $x$ and $y$, we need to retain the terms up to the third order. The zeroth order term can be dropped because $B_{r}$ and $B_{z}$ depend only on the derivatives of $\Psi$. The 
terms linear in $x$ and $z$ determine the magnetic field strength at $x=z=0$. We want both components of the magnetic field be zero and therefore, the linear terms in the expansion must be identically zero. As there are two of them, this requires adjustment of the two parameters of the external current system. For $B_{z}(x=0, z=0)=0$, the linear terms satisfy Eq. (11) identically, and it does not impose any additional constraints. Consider now quadratic terms. There are three of them, $\alpha_{1} x^{2}+\alpha_{2} x z+\alpha_{3} z^{2}$. Substituting this expression into Eq. (11), and taking into account that $B_{z}(x=0, z=0)=0$, we find that $\alpha_{3}=-\alpha_{1}$, so that $\Phi_{2}=\alpha_{1}\left(x^{2}-z^{2}\right)+\alpha_{2} x z$. As we want the linear terms in $\boldsymbol{B}$ vanish in the vicinity of the null point, we must put both $\alpha_{1}$ and $\alpha_{2}$ to be zero. This imposes two more constraints on the parameters of the external current loops. On the other hand, as has been mentioned above, two external current loops are characterized by six parameters. Thus far, we have imposed four constraints, two on the linear terms, and two on the quadratic terms, and, therefore, the system of two external loops is sufficient to reach the desired effect, with two parameters allowing for further manipulations of the field distribution (which is already made a second-order null).

To see the freedom that two remaining free parameters provide, consider the cubic terms on the expansion. There are four of them, $\Psi_{3}=\gamma_{1} x^{3}+\gamma_{2} x^{2} z+\gamma_{3} x z^{2}+\gamma_{4} z^{3}$. As both linear and quadratic terms in $\Psi$ are zero, substitution of this expression into Eq. (11) yields: $6 \gamma_{1} x+2 \gamma_{2} z+2 \gamma_{3} x+6 \gamma_{4} z=0$, i.e., $\gamma_{3}=-3 \gamma_{1}$, and $\gamma_{2}=-3 \gamma_{4}$. In other words,

$$
\Psi_{3}=\gamma_{1}\left(x^{3}-3 x z^{2}\right)+\gamma_{4}\left(z^{3}-3 x^{2} z\right)
$$

The ratio of two remaining free parameters, $\gamma_{1}$ and $\gamma_{4}$, determines the orientation of the snow-flake separatrix in the vicinity of the null-point. For example, if $\gamma_{1}=0$, then the separatrix is oriented as in the inset in Fig. 1a; if, on the other hand, $\gamma_{4}=0$, then the snowflake turns by 30 degrees, with other values of the ratio corresponding to the intermediate turns. The magnitude of the coefficients characterizes the strength of the hexapole component of the field.

Therefore, we have shown that creating a second-order null by a simple set of currents remains feasible in the toroidal geometry. Making it topologically stable can be reached by driving a somewhat higher current through the divertor coils, in the same way as discussed above for a "rectified" torus.

In summary: The snow-flake divertor can be realized by a simple set of coils situated at a significant distance from the null-point. It provides a much stronger expansion of the magnetic flux than the standard X-point divertor. Its magnetic configuration can be made topologically-stable by operating at the divertor currents slightly higher than those providing an exact second-order null. The connection length increases substantially compared to the X-point divertor. The magnetic field shearing near the null-point also becomes much higher. Potentially, all these properties may lead to a more efficient divertor, with substantially reduced heat loads on the divertor plates.

A disadvantage of this configuration is related to the somewhat higher (than standard) current in the divertor coils. Another problem is associated with that the flux becomes strongly broadened well above the null-point, making the geometrical connectivity of the snow-flake divertor with the main SOL easier than in the standard divertor. This may lead to the increased impurities flow to the main SOL.

The author is grateful to R.H. Cohen for helpful comments. Work performed for Department of Energy by UC LLNL under contract No. W-7405-Eng-48. 


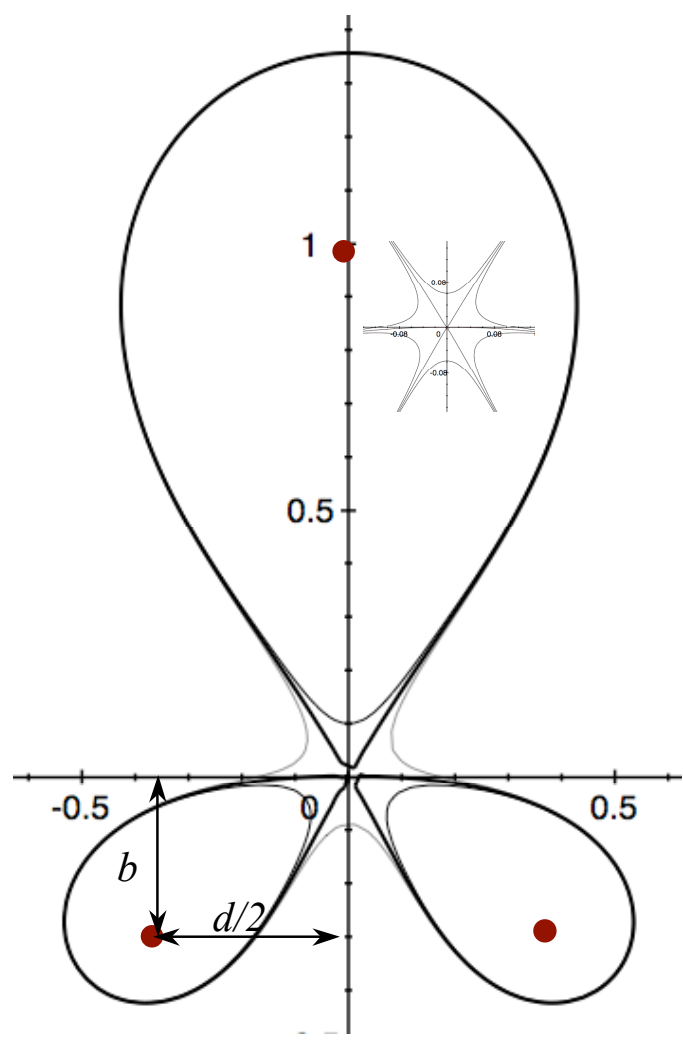

Fig. 1 A snow-flake divertor. The separatrix near the null point forms a characteristic hexagonal structure (an inset) reminiscent of a snow-flake. The distances are measured in the units of $a$ (which is the distance between the null-point and the conductor imitating the plasma current). The thick line represents the separatrix; a thin lines outside and inside the separatrix represent flux surfaces whose distance is $0.002 a$ from the separatrix in the equatorial plane (i.e., $1 \mathrm{~cm}$ for the device with $a=5 \mathrm{~m}$ ). At the scale of the figure, the $1 \mathrm{~cm}$ of distance in the main SOL is too small to be resolved, whereas the distance of the outer flux surface from the null-point is approximately $80 \mathrm{~cm}$. In other words, the broadening of the scrape-off-layer near the null-point is very large. 

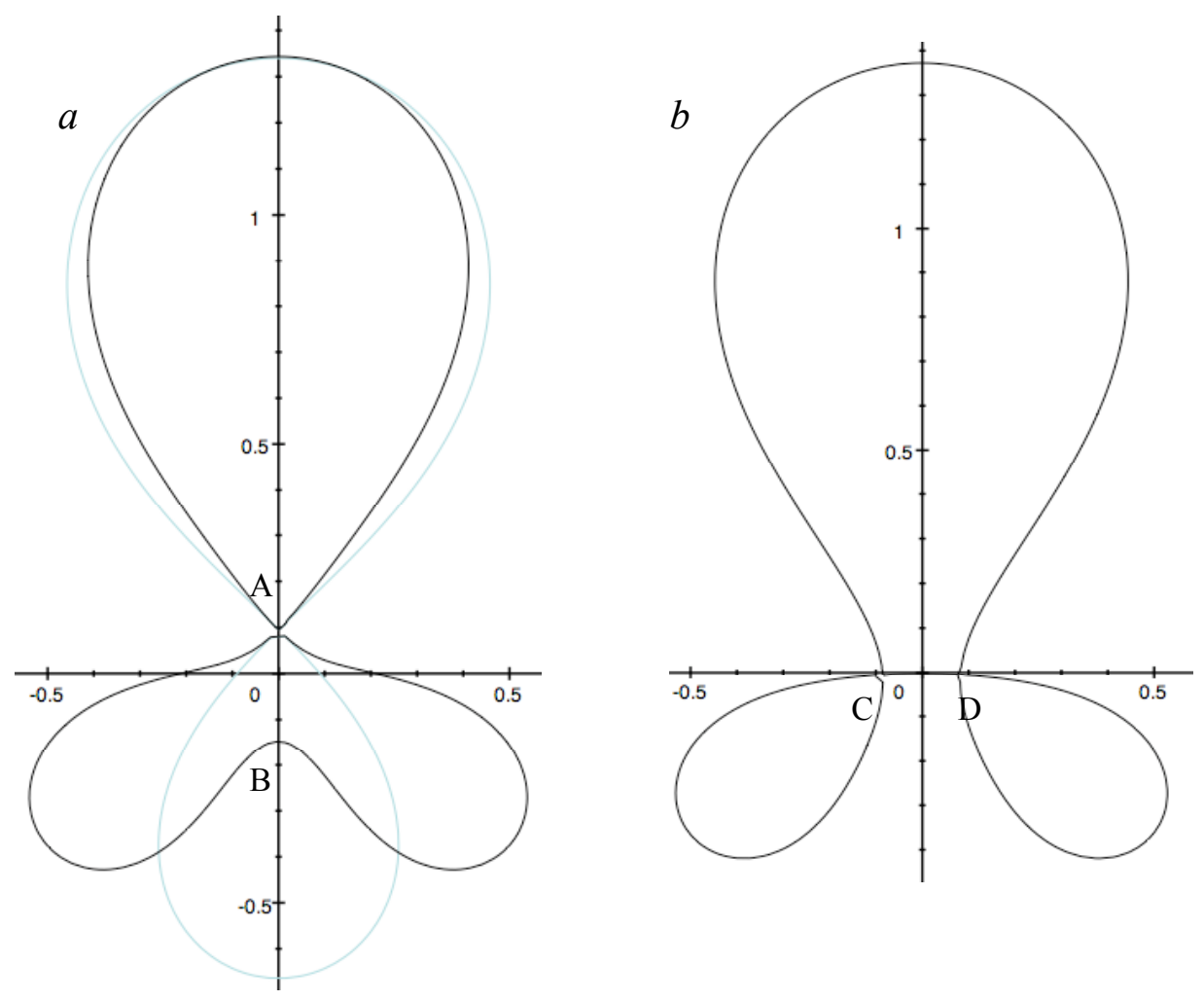

Fig. 2. The shape of the separatrix and two nearby flux surfaces for the case where the divertor current is five percent higher (a) and five percent lower (b) than the current $I_{d 0}$, Eq.(3). We call the first of them "snow-flake-plus" and the second "snow-flake-minus." Shown in light line in (a) is the separatrix of a "standard" X-point divertor with the upper part of the separatrix not much different from that of the snow-flake-plus divertor with $\varepsilon=0.05$. We compare these two configurations in terms of the flux expansion properties in Fig. 4. 


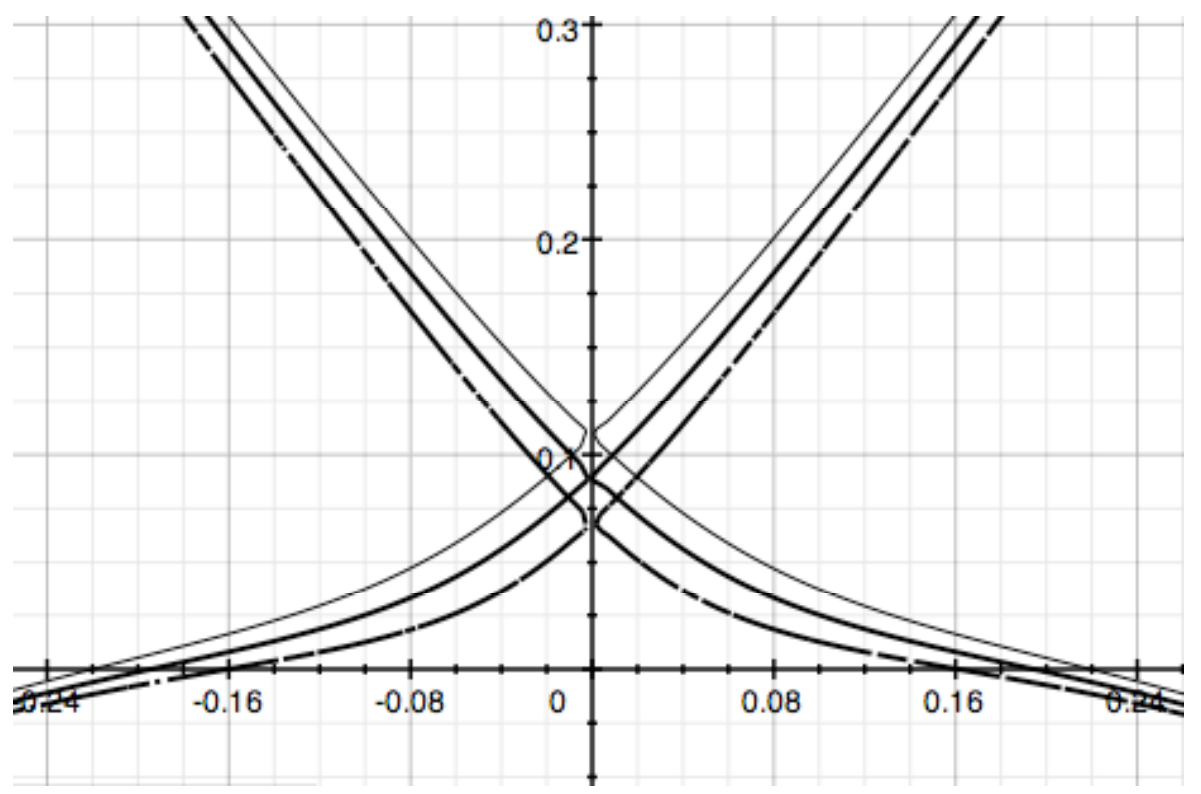

Fig. 3 Change of the separatrix shape in the vicinity of the null point with the change of the divertor current. The central (bold) line corresponds to a reference case of $\varepsilon=0.05$. The upper thin line corresponds to $\varepsilon=0.07$, whereas the lower thin curve corresponds to $\varepsilon=0.03$. With this variation, the motion of the separatrix is insignificant. 


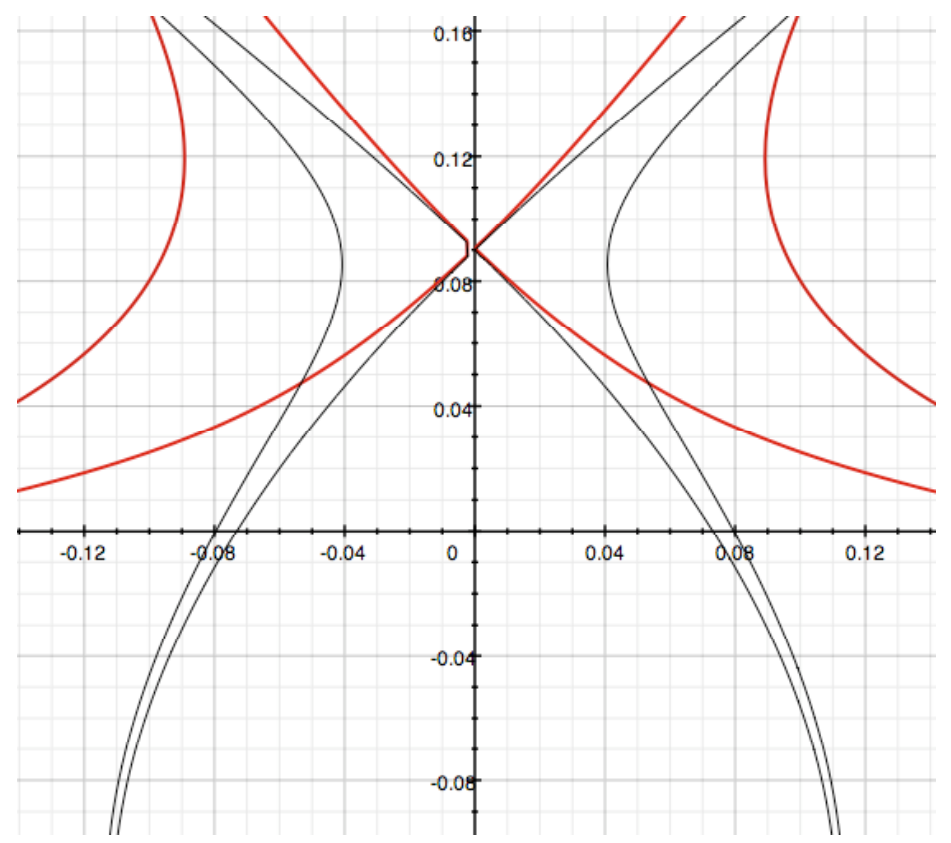

Fig. 4. The comparison of the SOL width near the null point in the modified snow-flake divertor and a standard X-point divertor. 


\section{References}

1. F. Najmabadi and the ARIES team, Fus. Eng. Des, 38, 3 (1997).

2. M. Kotschenreuther et al, 2004 IAEA Fusion Energy Conference (Vilamoura, Portugal, 1-6 November 2004) paper IC/P6-43.

3. Y. Andrew, N.C. Hawkes, M.G. O’Mullane, et al. Plasma Phys. Contr. Fus., 46, A87 (2004).

4. B. Labombard, J.E. Rice, A.E. Hubbard, et al. Phys. Plasmas, 12, 056111 (2005).

5. G.F. Counsell, A. Kirk, R.H. Cohen, et al. Nucl. Fusion 43 1197-1203 (2003).

6. R. Aymar, P. Barabasch, Y. Shimomura, et al. Plasma Phys. Contr. Fus., 44, 519 (2002).

7. D.Farina, R.Pozzoli, D.D. Ryutov. Nuclear Fusion, v.33, p.1315 (1993).

8. R.H. Cohen, D.D. Ryutov. Nucl. Fusion, 37, 621 (1997). 